\title{
Rational approximations to the Rogers-Ramanujan
} continued fraction

by

IEKATA ShIOKAWA (Yokohama, Japan)

1. Introduction. Let $F(\alpha)$ be defined by

$$
F(\alpha)=F(\alpha, x)=\sum_{n=0}^{\infty} \frac{\alpha^{n} x^{n^{2}}}{(1-x)\left(1-x^{2}\right) \ldots\left(1-x^{n}\right)} \quad(|x|<1) .
$$

Then $F(\alpha)$ satisfies

$$
F(\alpha)=F(\alpha x)+\alpha x F\left(\alpha x^{2}\right),
$$

so that $F(\alpha) / F(\alpha x)$ can be developed in the Rogers-Ramanujan continued fraction

$$
\frac{F(\alpha)}{F(\alpha x)}=1+\frac{\alpha x}{1}+\frac{\alpha x^{2}}{1}+\frac{\alpha x^{3}}{1}+\cdots
$$

In particular, by virtue of the Rogers-Ramanujan identities, we have

$$
\begin{aligned}
1+\frac{x}{1}+\frac{x^{2}}{1}+\ldots & =\frac{\sum_{n=0}^{\infty} \frac{x^{n^{2}}}{(1-x)\left(1-x^{2}\right) \ldots\left(1-x^{n}\right)}}{\sum_{n=0}^{\infty} \frac{x^{n^{2}+n}}{(1-x)\left(1-x^{2}\right) \ldots\left(1-x^{n}\right)}} \\
& =\prod_{n=0}^{\infty} \frac{\left(1-x^{5 n+2}\right)\left(1-x^{5 n+3}\right)}{\left(1-x^{5 n+1}\right)\left(1-x^{5 n+4}\right)} .
\end{aligned}
$$

(For details see for example [1], [5].) We put for brevity

$$
f(\alpha, x)=F(\alpha) / F(\alpha x)
$$

In 1971 Osgood [8], [9] proved that, if $a, b$, and $d$ are non-zero integers with $|d| \geqslant 2$, then, for any $\varepsilon>0$, there is a positive constant $q_{0}=q_{0}(a, b, d, \varepsilon)$ such that

$$
\left|f\left(\frac{a}{b}, \frac{1}{d}\right)-\frac{p}{q}\right|>q^{-2-q}
$$

for all integers $p, q\left(\geqslant q_{0}\right)$. 
For the values of the exponential function at rational points more precise results have been obtained (cf. Bundschuh [2], Durand [4], Mahler [7], Shiokawa [10]): If $a / b$ is a non-zero rational number, then there are explicit positive constants $B=B(a / b)$ and $C=C(a / b)$ such that

$$
\left|e^{a / b}-\frac{p}{q}\right|>C q^{-2-B / \log \log q}
$$

for all integers $p, q(\geqslant 3)$. Especially, Davis [3] proved that, if $b$ is a non-zero integer and

$$
C=\left\{\begin{array}{ll}
1 /|b| & \text { if } b \text { is even, } \\
1 /|4 b| & \text { otherwise }
\end{array} .\right.
$$

then, for any $\varepsilon>0$,

$$
\left|e^{2 / b}-\frac{p}{q}\right|<(C+\varepsilon) q^{-2} \frac{\log \log q}{\log q}
$$

for infinitely many integers $p, q$, while there is a positive constant $q_{0}=q_{0}(b, \varepsilon)$ such that

$$
\left|e^{2 / b}-\frac{p}{q}\right|>(C-\varepsilon) q^{-2} \frac{\log \log q}{\log q}
$$

for all integers $p, q\left(\geqslant q_{0}\right)$.

Comparing these results, we see that it would be interesting to replace, if possible, the $\varepsilon$ in Osgood's theorem stated above by a function of $q$. In this connection, we prove in this paper the following theorems.

Theorem 1. Let $a, b, c$, and $d$ be non-zero integers with

$$
|d|>|c|^{2} \text {. }
$$

Then $f(a / b, c / d)$ is an irrational number, and furthermore, there is a positive constant $C=C(a, b, c, d)$ such that

$$
\left|f\left(\frac{a}{b}, \frac{c}{d}\right)-\frac{p}{q}\right|>C q^{-2-2 A-B / \sqrt{\log q}}
$$

for all integers $p, q\left(\geqslant q_{0}\right)$, where

$$
A=\frac{\log |c|}{\log \left|d / c^{2}\right|}
$$

and

$$
B=\frac{\log \left|a^{2} d\right|-A \log \left|b / a^{2}\right|}{\sqrt{\log \left|d / c^{2}\right|}}
$$

COROLlARY. Let $a, b$, and $d$ be non-zero integers with $|d| \geqslant 2$. Then there is a positive constant $C=C(a, b, d)$ such that

$$
\left|f\left(\frac{a}{b}, \frac{1}{d}\right)-\frac{p}{q}\right|>C q^{-2-B / \sqrt{\log q}}
$$

for all integers $p, q(\geqslant 2)$, where

$$
B=\frac{\log \left|a^{2} d\right|}{\sqrt{\log |d|}}
$$

Theorem 1 is in a sense best possible since we have the following theorem:

THEOREM ${ }^{\hbar}$ 2. Let $a, b$, and $d$ be positive integers such that $(a, b)=1$, $d \geqslant 2$, and a divides $d$, and let

$$
C= \begin{cases}\sqrt{\frac{b}{a}} & \text { if }\left(\frac{a}{b}\right)^{2}>d \\ \sqrt{\frac{a}{b d}} & \text { otherwise }\end{cases}
$$

Then, for any $\varepsilon>0$,

$$
\left|f\left(\frac{a}{b}, \frac{1}{d}\right)-\frac{p}{q}\right|<(C+\varepsilon) q^{-2-\sqrt{\log d} / \sqrt{\log q}}
$$

for infinitely many integers $p, q(\geqslant 0)$, while there is a positive constant $q_{0}=q_{0}(a, b ; d, \varepsilon)$ such that

$$
\left|f\left(\frac{a}{b}, \frac{1}{d}\right)-\frac{p}{q}\right|>(C-\varepsilon) q^{-2-\sqrt{\log d} / \sqrt{\log q}}
$$

for all integers $p, q\left(\geqslant q_{0}\right)$

2. A lemma. We shall make use of the following lemma.

LEMMA. Let $a_{1}, a_{2}, a_{3}, \ldots$ be a sequence of real numbers such that

$$
\left|a_{n} a_{n+1}\right|>4 .(n \geqslant 1)
$$

and

$$
\sum_{n=1}^{\infty}\left|a_{n} a_{n+1}\right|^{-1}=\sigma<\infty
$$

Define as usual $p_{n}=a_{n} p_{n-1}+p_{n-2}, q_{n}=a_{n} q_{n-1}+q_{n-2}(n \geqslant 1)$ with $p_{0}=q_{-1}$ $=0, p_{\sim 1}=q_{0}=1$. Then $p_{n} /\left(a_{2} a_{3} \ldots a_{n}\right)$ and $q_{n} /\left(a_{1} a_{2} \ldots a_{n}\right)$ converge to finite non-zero limits, and they satisfy

$$
\begin{aligned}
& e^{-4 \sigma}<\left|p_{n} /\left(a_{2} a_{3} \ldots a_{n}\right)\right|<e^{2 \sigma}, \\
& e^{-4 \sigma}<\left|q_{n} /\left(a_{1} a_{2} \ldots, a_{n}\right)\right|<e^{2 \sigma},
\end{aligned}
$$


so that the continued fraction

$$
\frac{1}{a_{1}}+\frac{1}{a_{2}}+\frac{1}{a_{3}}+\cdots=\lim _{n \rightarrow \infty} \frac{p_{n}}{q_{n}}
$$

is convergent.

For the proof see [6], §4.4; [10].

To apply the lemma, we transform the continued fraction (1) by using the formula

$$
\frac{b_{1}}{1}+\frac{b_{2}}{1}+\frac{b_{3}}{1}+\ldots=\frac{1}{\frac{1}{b_{1}}}+\frac{1}{b_{1}}+\frac{1}{b_{2}}+\frac{1}{b_{1} b_{3}}+\frac{1}{b_{1} b_{3}}+\cdots
$$

(cf. [6], $(2,3,24))$ and obtain the regular continued fraction

$$
f(\alpha, x)=1+\frac{1}{a_{1}}+\frac{1}{a_{2}}+\frac{1}{a_{3}}+\cdots
$$

where

$$
a_{2 k-1}=\alpha^{-1} x^{-1}, \quad a_{2 k}=x^{-k} . \quad(k \geqslant 1) .
$$

We note here that

(4) $a_{1} a_{2} \ldots a_{2 k-1}=\alpha^{-k} x^{-k^{2}}, \quad a_{1} a_{2} \ldots a_{2 k} \alpha^{-k} x^{-k^{2}-k} \quad(k \geqslant(1)$, and hence

$$
\log \left|a_{1} a_{2} \ldots a_{n}\right|=-\frac{1}{4} n^{2} \log |x|-\frac{1}{2} n \log |\alpha x|+O(1)
$$

3. Proof of Theorem 1. Let $\alpha=a / b$ and $x=c / d$ 'be as in Theorem 1 . Then $a_{n}$, and hence, $p_{n}, q_{n}$ are rational numbers for which $d_{n} p_{n}, d_{n} q_{n}$ are integers for all $n \geqslant 1$, where

so that

$$
d_{2 k-1}=\left|a^{k} c^{k^{2}}\right|, \quad d_{2 k}=\left|a^{k} c^{k^{2}+k}\right|
$$

$$
\log d_{n}=\frac{1}{4} n \log |c|+\frac{1}{2} n \log |a c|+O(1)
$$

Here and in what follows constants implied in $O$-symbols as well as positive constants $m, n_{0}, c_{0}, c_{1}, \ldots$ depend possibly on $a, b, c, d$ (and $\varepsilon$ in Section 4).

Since $a_{n} a_{n+1}=\alpha^{-1} x^{-n}(n \geqslant 1)$ with $|x|<1$, the series $\sum_{n=1}^{\infty}\left(a_{n} a_{n+1}\right)^{-1}$ is absolutely convergent and there exists an integer $m \geqslant 1$ such that $\left|a_{n} a_{n+1}\right|>4(n \geqslant m)$. We may thus apply the lemma and find that the
continued fraction

$$
\frac{1}{a_{n+1}}+\frac{1}{a_{n+2}}+\frac{1}{a_{n+3}}+\cdots=\theta_{n}, \quad \text { say, }
$$

is convergent for each $n \geqslant m$ and

$$
\begin{aligned}
& e^{-6 \sigma}<\left|a_{n+k+1} \theta_{n+k}\right|<e^{6 \sigma} \\
& e^{-6 \sigma}<\left|a_{n+k+1} q_{n, k} / q_{n, k+1}\right|<e^{6 \sigma} \quad(n \geqslant m, k \geqslant 1),
\end{aligned}
$$

where $p_{n, k} / q_{n, k}$ is the $k$ th convergent of the continued fraction (7) and $\sigma=\sum_{n=1}^{\infty}\left|a_{n} a_{n+1}\right|^{-1}$. Hence

$$
\left|\theta_{n}-\frac{p_{n, k}}{q_{n, k}}\right|=\frac{1}{\left|q_{n, k}\left(q_{n, k+1}+\theta_{n+k+1} q_{n, k}\right)\right|}<\frac{2}{\left|q_{n, k}^{2} a_{n+k+1}\right|}
$$

for all sufficiently large $k$. But using again the lemma with (5) and (6), we get

$$
\frac{\log \left|q_{n, k}^{2} a_{n+k+1}\right|}{\log \left|d_{n+k+1} q_{n, k}\right|}>2-\frac{2 \log |c|}{\log |d|}-\frac{C_{0}}{k}
$$

so that, for any $\varepsilon>0$,

$$
\left|\theta_{n}-\frac{d_{n+k} p_{n, k}}{d_{n+k} q_{n, k}}\right|<\cdot\left|d_{n+k} q_{n, k}\right|^{-2+2(\log |c|) / \log |d|+\varepsilon}
$$

for all sufficiently large $k$. This establishes the irrationality of $\theta_{n}(n \geqslant m)$, since $d_{n+k} p_{n, k}, d_{n+k} q_{n, k}$ are integers and $2(\log |c|) / \log |d|<1$ by (2).

Now we may assume $p_{m} q_{m} \neq 0$, since at least one of $p_{n-1} q_{n-1}, p_{n} q_{n}$ is different from zero, because $a_{n} \neq 0(n \geqslant 1)$. It follows from the formula $p_{n}=p_{m} q_{m, n-m}+p_{m-1} p_{m, n-m}, q_{n}=q_{m} q_{m, n-m}+q_{m-1} p_{m, n-m}$ that

$$
\begin{aligned}
& \frac{p_{n}}{a_{2} a_{3} \ldots a_{n}}=\frac{p_{m}}{a_{2} a_{3} \ldots a_{n}} \frac{q_{m, n-m}}{a_{m+1} \ldots a_{n}}\left(1+\frac{p_{m-1}}{p_{m}} \frac{p_{m, n-m}}{q_{m, n-m}}\right), \\
& \frac{q_{n}}{a_{1} a_{2} \ldots a_{n}}=\frac{q_{m}}{a_{1} a_{2} \ldots a_{n}} \frac{q_{m, n-m}}{a_{m+1} \ldots a_{n}}\left(1+\frac{q_{m-1}}{q_{m}} \frac{p_{m, n-m}}{q_{m, n-m}}\right) .
\end{aligned}
$$

By the lemma, quantities on the right-hand side above converge as $n \rightarrow \infty$ to finite limits which are different from zero, because of the fact that $\theta_{m}$ is irrational and $p_{m} q_{m} \neq 0$. Hence the continued fraction $\left(1^{\prime}\right)$ converges to $f(a / b, c / d)$, which, as is easily seen, is also irrational. Thus we have, using (5),

$$
\log \left|q_{n}\right|=\frac{n^{2}}{4} \log \left|\frac{d}{c}\right|+\frac{n}{2} \log \left|\frac{b d}{a c}\right|+O(1)
$$

and so, using (6),

$$
\log \left|\frac{q_{n+1}}{d_{n+1}}\right|-\log \left|\frac{q_{n}}{d_{n}}\right|=\frac{n}{2} \log \left|\frac{d}{c^{2}}\right|+O(1) .
$$

Hence, noticing (2) and (8), we can choose $n_{0} \geqslant m$ such that

(11) $\quad\left|\theta_{n}\right|<1 / 2, \quad\left|q_{n-1}\right|<\left|q_{n}\right|, \quad\left|q_{n-1} / d_{n-1}\right|<\left|q_{n} / d_{n}\right| \quad\left(n \geqslant n_{0}\right)$. 
Now let $p, q$ be given non-zero integers. We may assume that $\left|q_{n_{0}} / d_{n_{0}}\right|$ $<4 q$. Then by $(10)$ and (11), there is an integer $n=n(q) \geqslant n_{0}$ such that

$$
\left|q_{n-1} / d_{n-1}\right| \leqslant 4 q<\left|q_{n} / d_{n}\right| \text {. }
$$

By virtue of the formula $p_{n} q_{n-1}-p_{n-1} q_{n}= \pm 1$, at least one of $p_{n-1} q$ $-q_{n-1} p, p_{n} q-q_{n} p$ is different from zero. Assume first that $p_{n} q-q_{n} p \neq 0$. Then we have

$$
d_{n} q_{n}\left(f\left(\frac{a}{b}, \frac{c}{d}\right)-\frac{p}{q}\right)=\frac{d_{n}\left(p_{n} q-q_{n} p\right)}{q}+d_{n}\left(q_{n} f\left(\frac{a}{b}, \frac{c}{d}\right)-p_{n}\right),
$$

where $\left|d_{n}\left(p_{n} q-q_{n} p\right)\right| \geqslant 1$ and

$$
\left|d_{n}\left(q_{n} f\left(\frac{a}{b}, \frac{c}{d}\right)-p_{n}\right)\right|=\frac{d_{n}}{\left|q_{n+1}+\theta_{n+1} q_{n}\right|} \leqslant \frac{2 d_{n}}{\left|q_{n}\right|}<\frac{1}{2 q},
$$

so that

$$
\left|f\left(\frac{a}{b}, \frac{c}{d}\right)-\frac{p}{q}\right|>\frac{1}{2} q^{-1-\left(\log \left|d_{n} q_{n}\right|\right) / \log q}
$$

The same inequality will be obtained also in the case of $p_{n-1} q-q_{n-1} p \neq 0$.

It remains to estimate $\left|d_{n} q_{n}\right|$ from above in terms of $q$. Combining (3), (6), (9), and (12), we get

$$
\begin{aligned}
\log \left|d_{n} q_{n}\right| & \leqslant \log q+\log \left(d_{n-1} d_{n}\right)+\log \left|a_{n}\right|+C_{1} \\
\therefore & \leqslant \log q+\frac{1}{2} n^{2} \log |c|+\frac{1}{2} n \log \left|a^{2} d\right|+C_{2} .
\end{aligned}
$$

Here it follows from (12) with (6) and (9) that

$$
\frac{n^{2}}{4} \log \left|\frac{d}{c^{2}}\right|+\frac{n}{2} \log \left|\frac{b}{a^{2}}\right|-C_{3}<\log q<\frac{n^{2}}{4} \log \left|\frac{d}{c^{2}}\right|+\frac{n}{2} \log \left|\frac{b d}{a^{2} c^{2}}\right|+C_{4},
$$

so that

$$
n=2 \sqrt{\log q} / \sqrt{\log \left|d / c^{2}\right|}+o(1)
$$

and hence

$$
n^{2} \leqslant \frac{4 \log q}{\log \left|c / d^{2}\right|}-\frac{4 \sqrt{\log q} \log \left|b / a^{2}\right|}{\sqrt{\log \left|c / d^{2}\right|} \log \left|c / d^{2}\right|}+C_{5} .
$$

Therefore, we obtain

$$
\frac{\log \left|d_{n} q_{n}\right|}{\log q}<1+A+\frac{B}{\sqrt{\log q}}
$$

which together with (13) leads to Theorem 1.
4. Proof of Theorem 2. Let $\alpha=a / b$ and $x=1 / d$ as in Theorem 2. Then $f(a / b, 1 / d)$ can be developed in the regular continued fraction

$$
f\left(\frac{a}{b}, \frac{1}{d}\right)=1+\frac{1}{\frac{b}{a} d+d+\frac{1}{a} d^{2}+d^{2}+\ldots}+\frac{1}{+\frac{b}{a} d^{n}+d^{n}+} \frac{1}{}
$$

whose partial denomunators are positive integers, so that its convergents $p_{n} / q_{n}$ $(n \geqslant 1)$ are just all the best approximations to $f(a / b, 1 / d)$. Thus we have only to estimate

$$
\left|f\left(\frac{a}{b}, \frac{1}{d}\right)-\frac{p_{n}}{q_{n}}\right|=\frac{1}{\left|1+\frac{\theta_{n+1}}{a_{n+1}}+\frac{q_{n-1}}{a_{n+1} q_{n}}\right|} \frac{1}{\left|q_{n}^{2} a_{n+1}\right|} .
$$

We note first that

$$
\lim _{n \rightarrow \infty} \theta_{n+1} / a_{n+1}=\lim _{n \rightarrow \infty} q_{n-1} /\left(q_{n} a_{n+1}\right)=0 .
$$

If $n=2 k$, then by (3)

$$
\log a_{2 k+1}=k \log d+\log (d b / a) .
$$

But by (4)

$$
k=\frac{\sqrt{\log q_{2 k}}}{\sqrt{\log d}}-\frac{\log (d b / a)}{2 \log d}+o(1),
$$

and hence

$$
\frac{\log a_{2 k+1}}{\log q_{2 k}} \geqslant \frac{\sqrt{\log d}}{\sqrt{\log q_{2 k}}}+\frac{1}{2} \log (d b / a)+o(1) .
$$

Similarly, we get

$$
\frac{\log a_{2 k}}{\log q_{2 k-1}} \geqslant \frac{\sqrt{\log d}}{\sqrt{\log q_{2 k-1}}}+\frac{1}{2} \log (a / b)+o(1) .
$$

(14) together with these estimates yields Theorem 2 .

\section{References}

[1] G. E. Andrews, The Theory of Partitions, Addison-Wesley, London 1976.

2] P. Bundschuh, Irrationalitütsmasse fiur $e^{u}, a \neq 0$ rational oder Lioutille-Zahl, Math Ann. 192 (1971), pp. 229-242.

[3] C. S. Davis, Rational approximations to e, J. Austral. Math. Soc. (ser. A) 25 (1978), pp. $497-502$ 
[4] A. Durand, Simultaneous Diophantine approximations and Hermite's method, Bull. Austral. Math. Soc. 21 (1980), pp: 463-470.

[5] G. H. Hardy, Ramanujan, 3rd. ed., Chelsea, New York 1987.

[6] W. B. Jones and W. J. Thron, Continued Fractions: Analytic Theory and Applications, Addison-Wesley, London 1980.

[7] K. Mahler, On rational approximations of the exponential function at rational points, Bull. Austral. Math. Soc. 10 (1974), pp. 325-335.

[8] C. F. Osgood, On the Diophantine approximation of values of functions satisfying certain linear q-difference equations, J. Number Theory 3 (1971), pp. 159-177.

[9] - The Diophantine approximation of certain continued fractions, Proc. Amer. Math. Soc. 3 (1972), pp. 1-7.

[10] I. Shiokawa, Rational approximations to the values of certain hypergeometric functions, in Number Theory and Combinatorics, Japan 1984, World Scientific Publ. Co., Singapore 1985

\section{DEPARTMENT OF MATHEMATICS}

KEIO UNIVERSITY

\section{Poincaré series and Kloosterman sums for $\operatorname{SL}(3, Z)$}

by

Daniel Bump (Stanford, Cal.), Solomon Friedberg (Santa Cruz, Cal.) and Dorian Goldfeld* (New York, N. Y.)

\section{CONTENTS}

\author{
1. Introduction \\ 2. Poincaré series \\ 3. Invariants of $\Gamma_{\infty} \backslash \Gamma$ and Brahat decompositions \\ 4. $\mathrm{SL}(3, Z)$ Kloosterman sums \\ 5. Fourier expansion of Poincaré series \\ 6. Spectral decomposition of Poincaré series, cuspidal contribution \\ 7. Continuous spectrum \\ 8. The Poincaré series associated with a Bruhat cell \\ Appendix: Estimation of SL $(3, Z)$ Kloosterman sums by Michael Larsen
}

1. Introduction. For $z=x+i y, y>0$, let

$$
\Delta(z)=e^{2 \pi i z} \prod_{n=1}^{\infty}\left(1-e^{2 \pi i n z}\right)^{24}=\sum_{n=1}^{\infty} \tau(n) e^{2 \pi i n z}
$$

by the Ramanujan cusp form of weight 12 . Ramanujan conjectured that

$$
\tau(n)=O\left(n^{11 / 2+\varepsilon}\right)
$$

for any $\varepsilon>0$. This coniecture was proved by Deligne [2] in 1974. Actually, Deligne proved the more general result (Petersson conjecture) that

$$
a(n)=O\left(n^{(k-1) / 2+e}\right)
$$

where $a(n)$ is the $n$th Fourier coefficient of a holomorphic cusp form of weight $k$ associated to a congruence subgroup of $\operatorname{SL}(2, Z)$.

Ramanujan's conjecture can be generalized to non-holomorphic cusp forms (Maass wave forms) associated to arithmetic discrete subgroups of $\mathrm{GL}(r, \boldsymbol{R}), r \geqslant 2$, and in this form, the conjecture is still open even for $r=2$. We now briefly describe the generalized Ramanujan conjecture.

* This author would like to acknowledge supports from the Vaughn Foundation and also from the NSF grant DMF-8502787. 